\title{
Decoherence and Loschmidt echoes: Quantum against Classical
}

\author{
Tomaž Prosen and Marko Žnidarič \\ Department of Physics, Faculty of Mathematics and Physics, \\ University of Ljubljana, Jadranska 19, SI-1000 Ljubljana, Slovenia \\ JUNE 1, 2005; Received on 20 May, 2005
}

\begin{abstract}
Some recent theoretical results on the stability of quantum dynamics against perturbations of the Hamiltonian - the so-called Loschmidt echoes and their relation to various decoherence measures are reviewed. We show that the representation of Loschmidt echoes in terms of the Wigner function can explain some seemingly paradoxical behavior of the quantum-classical correspondence.
\end{abstract}

\section{INTRODUCTION}

Studies of stability of quantum time evolution with respect to small variation in the Hamiltonian have attracted considerable interest in recent years [1-5]. Quite remarkable is the fact that the problem has been practically intact until 2001, with exception of experimental studies of the group of Pastawski [6] in the mid 1990's. However, one has to mention the pioneering paper of Peres in 1984 [7], where he poses the question and suggests the overlap between quantum states of two nearby time-evolutions as a signature of chaos in quantum mechanics. However, as one understands today, his conjecture, that this overlap - now called the fidelity - decays faster for classically chaotic than for classically regular dynamics, generally fails. The fidelity is also known as the Loschmidt echo since it can be equivalently interpreted, due to unitarity of quantum time evolution, in terms of an overlap between the initial state, and the state obtained after composing forward time evolution with the slightly perturbed backward evolution.

The behavior of fidelity is also intimately connected to the scale of time $t$, perturbation strength $\delta$, and effective Planck constant $\hbar$. There are many different regimes and one has to be careful when discussing general results. For example, in classically chaotic systems there are basically two main regimes, depending on the non-dimensional ratio $\sigma=\delta / \hbar$. If $\sigma>1$, then the drop of fidelity is very fast (within the so-called Ehrenfest time-scale), so one can use strict quantum-classical correspondence and direct semi-classical theories in order to explain fidelity decay. For classically chaotic systems, it has been found that fidelity exhibits perturbation independent exponential decay $\propto e^{-\lambda t}$ where $\lambda$ is simply the classical Lyapunov exponent [1]. In the opposite regime $\sigma<1$, one can use quantum time-dependent perturbation theory in order to derive the fidelity decay. Again, for chaotic systems, one usually finds exponential decay [2-4] $\propto e^{-\Gamma t}$ with the rate $\Gamma \propto \delta^{2}$, which is usually referred to as a Fermi golden rule regime.

It is however interesting to note that the rate $\Gamma$ is typically smaller the stronger the corresponding classical chaos (or rate of dynamical mixing). This is apparently in contrast to any classical intuition. Therefore there is no direct correspondence between quantum fidelity and the corresponding classical object, i.e. the classical fidelity, beyond the Ehrenfest time.

In this paper we make a detailed illustration of the breakup of quantum-classical correspondence for fidelities (section III), after making a short review of the linear response the- ory of quantum fidelity in section II. In section IV we quote a quantitative relation between quantum fidelity and a measure of decoherence and conclude.

\section{LINEAR RESPONSE THEORY OF QUANTUM FIDELITY}

We consider a general quantum Hamilton operator $H_{\delta}(t)$ which may depend explicitly on time $t$, as well as on some external parameter $\delta$, like magnetic field, shape of potential well etc. Without essential loss of generality we shall assume that the parameter dependence is linear, namely

$$
H_{\delta}(t)=H_{0}(t)+\delta V(t)
$$

Let $U_{\delta}(t)$ be the propagator

$$
U_{\delta}(t)=\hat{T} \exp \left(-\frac{\mathrm{i}}{\hbar}_{0}^{\mathrm{Z}}{ }^{t} \mathrm{~d} t^{\prime} H_{\delta}\left(t^{\prime}\right)\right)
$$

where $\hat{T}$ designates the time ordering.

If $|\psi\rangle$ is some arbitrary initial state, then we may define the time evolution, depending on the time variable $t$ as well as on the perturbation parameter $\delta$ of the Hamiltonian

$$
\left|\psi_{\delta}(t)\right\rangle=U_{\delta}(t)|\psi\rangle
$$

Having the tools defined above, fidelity amplitude, with respect to the unperturbed evolution $U_{0}(t)$, is defined as the overlap of the perturbed and unperturbed time-evolving states

$$
f_{\delta}(t)=\left\langle\psi_{0}(t) \mid \psi_{\delta}(t)\right\rangle=\left\langle\psi\left|U_{0}(-t) U_{\delta}(t)\right| \psi\right\rangle .
$$

In the following we shall write the expectation value in a given initial state simply as $\langle A\rangle=\langle\psi|A| \psi\rangle$. Fidelity amplitude may now be compactly written in terms of expectation value of the so-called echo-operator

$$
M_{\delta}(t)=U_{0}(-t) U_{\delta}(t),
$$

namely

$$
f_{\delta}(t)=\left\langle M_{\delta}(t)\right\rangle
$$

Usually one studies fidelity $F_{\delta}(t)$, which is simply the absolute value square of the fidelity amplitude,

$$
F_{\delta}(t)=\left|f_{\delta}(t)\right|^{2} .
$$


We note that one may also use a non-pure initial state, namely a statistical mixture described by a density matrix $\rho$, with $\langle A\rangle=\operatorname{tr} \rho A$. Writing the perturbation operator in the interaction picture

$$
\tilde{V}(t)=U_{0}(-t) V(t) U_{0}(t)
$$

one can straightforwardly check, by differentiating Eq. (5), that the echo-operator solves the Heisenberg equation

$$
\frac{\mathrm{d}}{\mathrm{d} t} M_{\delta}(t)=-\frac{\mathrm{i}}{\hbar}\left[\delta \tilde{V}(t), M_{\delta}(t)\right]
$$

with the (effective) Hamiltonian $\delta \tilde{V}(t)$. Of course, the echooperator is nothing else but a propagator in the interaction picture, which can be written in terms of a formal solution of Eq. (9)

$$
M_{\delta}(t)=\hat{T} \exp \left(-\frac{\mathrm{i}}{\hbar} \delta_{0}^{\mathrm{Z}} \mathrm{d} t^{\prime} \tilde{V}\left(t^{\prime}\right)\right)
$$

where $\hat{T}$ denotes a time-ordering. The computation of fidelity becomes rather straightforward if one plugs the expression for the echo-operator (10) into the definition (6). In particular, this approach is ideally suited for a perturbative treatment when the perturbation strength $\delta$ can be considered as a small parameter. One can write explicit Born series for the echo operator, which has an infinite radius of convergence provided only that the perturbation operator $V(t)$ is a bounded, uniformly for all $t$ :

$$
\begin{aligned}
M_{\delta}(t) & =\mathbf{1}+\sum_{m=1}^{\infty} \frac{(-\mathrm{i} \delta)^{m}{ }^{\mathrm{Z}}{ }_{t}}{\hbar^{m} m !}{ }_{0} \mathrm{~d} t_{1} \mathrm{~d} t_{2} \cdots \mathrm{d} t_{m} \times \\
& \times \hat{T} \tilde{V}\left(t_{1}\right) \tilde{V}\left(t_{2}\right) \cdots \tilde{V}\left(t_{m}\right) .
\end{aligned}
$$

If we truncate the above series at second order, $m=2$, and plug into (7), we obtain

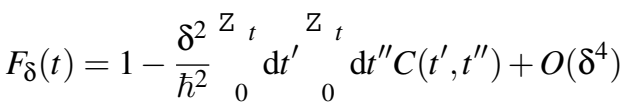

where

$$
C\left(t^{\prime}, t^{\prime \prime}\right)=\left\langle\tilde{V}\left(t^{\prime}\right) \tilde{V}\left(t^{\prime \prime}\right)\right\rangle-\left\langle\tilde{V}\left(t^{\prime}\right)\right\rangle\left\langle\tilde{V}\left(t^{\prime \prime}\right)\right\rangle,
$$

is just a 2-point time-correlation function of the perturbation. High-fidelity approximation (12) is usually called linear response expression of fidelity.

We stress that the validity of the linear response formula (12) is by no means restricted to short times. The only condition is the high-fidelity namely, $\eta:=1-F_{\delta}(t)$ has to be small, and the error of linear approximation is typically of order $O\left(\eta^{2}\right)=O\left(\delta^{4}\right)$.

Note that linear-response formula (12) already establishes a very important physical properties of the quantum echodynamics. One should observe an intimate connection between fidelity decay and temporal-correlation decay, namely faster decay of correlation function $C\left(t^{\prime}, t^{\prime \prime}\right)$, as $\left|t^{\prime}-t^{\prime \prime}\right|$ grows, implies slower decay of fidelity and vice versa. For example, for chaotic quantum systems in a semi-classical regime, whose classical limit is chaotic and fast mixing, such that $C\left(t^{\prime}, t^{\prime \prime}\right)$ decays faster than const $/\left|t^{\prime}-t^{\prime \prime}\right|$, the fidelity is expected to decay as a linear function of time $F_{\delta}(t)=1-$ const $\delta^{2} t$, whereas for regular systems, with integrable classical limit, $C\left(t^{\prime}, t^{\prime \prime}\right)$ is expected to have oscillating behavior with non-vanishing time average, hence fidelity is expected to decay as quadratic function of time $F_{\delta}(t)=1-\operatorname{const} \delta^{2} t^{2}$. This implies one of the seemingly paradoxical conclusions of our analysis, namely that the fidelity of regular dynamics decays faster than of the chaotic one.

\section{WIGNER REPRESENTATION OF FIDELITY, CLASSICAL FIDELITY AND QUANTUM CLASSICAL CORRESPONDENCE}

Now we wish to use a concept of quantum mechanics in phase space in terms of Wigner functions in oder to give a clear illustration of the behaviour of quantum fidelity, as well as its classical corresponence.

Let us assume that our system has $d$-degrees of freedom, which in the classical phase space can be represented by a point $\vec{x}=(\vec{q}, \vec{p})$ in $2 d$-dimensional Euclidean space. Using the Wigner function of the pure state $\psi(\vec{q})=\langle\vec{q} \mid \psi\rangle$

$$
W_{\psi}(\vec{q}, \vec{p})=(2 \pi \hbar)^{-d} \mathrm{~d}^{d} \vec{r} \psi^{*}(\vec{q}-\vec{r} / 2) \psi(\vec{q}+\vec{r} / 2) e^{i \vec{r} \cdot \vec{p} / \hbar},
$$

which for two states $|\psi\rangle,|\eta\rangle$ satisfies the principal property

$$
|\langle\eta \mid \psi\rangle|^{2}=(2 \pi \hbar)^{d} \quad \mathrm{~d}^{2 d} \vec{x} W_{\eta}(\vec{x}) W_{\psi}(\vec{x})
$$

one can write the fidelity simply in terms of an overlap of time-evolving Wigner functions

$$
\begin{aligned}
F_{\delta}(t) & =(2 \pi \hbar)^{d} \mathrm{~d}^{2 d} \vec{x} W_{U(t) \psi}(\vec{x}) W_{U_{\delta}(t) \psi}(\vec{x}) \\
& =(2 \pi \hbar)^{d} \mathrm{~d}^{2 d} \vec{x} W_{\psi}(\vec{x}) W_{M_{\delta}(t) \psi}(\vec{x})
\end{aligned}
$$

The second identity trivially follows from the unitarity of quantum time evolutions. Since, due to (15), ${ }_{\mathrm{R}}$ for pure states the Wigner function is $L^{2}$ normalized to $\mathrm{d}^{2 d} \vec{x} W_{\psi}^{2}(\vec{x})=$ $(2 \pi \hbar)^{-d}$, one can write the deviation of fidelity from 1 as

$$
\begin{aligned}
& 1-F_{\delta}(t)= \\
& (2 \pi \hbar)^{d} \quad \mathrm{~d}^{2 d \vec{x}} W_{U(t) \psi}(\vec{x})\left[W_{U(t) \psi}(\vec{x})-W_{U_{\delta}(t) \psi}(\vec{x})\right]
\end{aligned}
$$

If $h_{\delta}(\vec{x}, t)$ is the Weyl-symbol corresponding to the Hamiltonian operator $H_{\delta}(t)$, then the evolution of the Wigner function is governed in terms of the Moyal equation

$$
\frac{\mathrm{d}}{\mathrm{d} t} W_{U_{\delta}(t) \psi}(\vec{x})=\left\{h_{\delta}(\vec{x}, t), W_{U_{\delta}(t) \psi}(\vec{x})\right\}_{\mathrm{MB}}
$$

where

$$
\begin{aligned}
& \{a(\vec{x}), b(\vec{x})\}_{\mathrm{MB}}= \\
& -\frac{2}{\hbar} \sin \left[\frac{\hbar}{2}\left(\frac{\partial^{(a)}}{\partial \vec{p}} \cdot \frac{\partial^{(b)}}{\partial \vec{q}}-\frac{\partial^{(a)}}{\partial \vec{q}} \cdot \frac{\partial^{(b)}}{\partial \vec{p}}\right)\right] a(\vec{x}) b(\vec{x})
\end{aligned}
$$


is the Moyal bracket. The Moyal equation is a good starting point for understanding details of quantum classical correspondence, as expansion of Moyal bracket in powers of $\hbar$ gives $\{a(\vec{x}), b(\vec{x})\}_{\mathrm{MB}}=\{a(\vec{x}), b(\vec{x})\}_{\mathrm{PB}}+O\left(\hbar^{2}\right)$ where $\{\bullet, \bullet\}\}_{\mathrm{PB}}$ is just a classical Poisson bracket. Starting with a state having positive Wigner function, $W_{\psi}(\vec{x}) \geq 0$ for all $\vec{x}$, one can thus approximate $W_{U_{\delta}(t) \psi}(\vec{x})$ with a classical density $\rho_{\delta}(\vec{x}, t)$ solving the Liouville equation

$$
\frac{\mathrm{d}}{\mathrm{d} t} \rho_{\delta}(\vec{x}, t)=\left\{h_{\delta}(\vec{x}, t), \rho(\vec{x}, t)\right\}_{\mathrm{PB}}
$$

and satisfying the initial condition $\rho_{\delta}(\vec{x}, 0):=W_{\psi}(\vec{x})$. For chaotic systems, classical densities approximate the Wigner function for times up to $t^{*} \propto \log (1 / \hbar) / \lambda$ where $\lambda$ is the classical Lyapunov exponent. Such a time-scale $t^{*}$ is sometimes known as the Ehrenfest time.

Actually, one can write (or define!) the classical corresponding quantity to fidelity in terms of the classical densities $\rho_{\delta}(\vec{x}, t)$, simply by substituting Wigner functions by classical densities, and using the appropriate $L^{2}$ normalizations,

$$
\begin{aligned}
F_{\delta}^{\mathrm{cl}}(t) & =\frac{\mathrm{d} \vec{x} \rho_{0}(\vec{x}, t) \rho_{\delta}(\vec{x}, t)}{\mathrm{d} \vec{x} \rho_{0}^{2}(\vec{x}, 0)} \\
& =\frac{\mathrm{d} \vec{x} \rho_{0}(\vec{x}, 0) \rho_{0}\left(\vec{x}_{\mathrm{E}}(\vec{x}, t), 0\right)}{\mathrm{d} \vec{x} \rho_{0}^{2}(\vec{x}, 0)}
\end{aligned}
$$

We note that $\rho_{0}\left(\vec{x}_{\mathrm{E}}(\vec{x}, t), 0\right)$ is the classical density governed by the classical echo-dynamics, composing perturbed Liuovillian dynamics with an unperturbed backward Liouvillian dynamics, and $\vec{x}_{\mathrm{E}}(\vec{x}, t)$ are its characteristics echo-trajectories. Note an interesting fact [8], namely that the echo-flow $\vec{x}_{\mathrm{E}}(\vec{x}, t)$ satisfies canonical Hamilton's equations with explicitly time-dependent classical "Hamiltonian" $h_{\mathrm{E}}\left(\vec{x}_{0}, t\right)=\delta v\left(\vec{x}\left(\vec{x}_{0}, t\right), t\right)$ where $v(\vec{x}, t)$ is the Weyl-symbol of the perturbation part of the Hamiltonian in the classical interaction picture. For strictly hyperbolic, i.e. Anosov, classical systems, classical fidelity $F_{\delta}^{\mathrm{cl}}(t)$ has been shown to exhibit a cascade of exponential decays with exponents given by the sums of the leading classical Lyapunov exponents. For systems of one-degrees of freedom (or two degrees of freedom if the system is explicitly time-independent), this is always the one (non-negative) Lyapunov exponent.

Based on the quantum-classical correspondence we expect $F_{\delta}(t)=F_{\delta}^{\mathrm{cl}}(t)$ for $t \ll t^{*}$ due to point-wise agreement between Wigner functions and classical densities. After the Ehrenfest time, the quantum Wigner function starts to develop negative values and becomes an increasingly erratic object [9], in particular for non-integrable systems. As a consequence, at this time scale quantum fidelity $F_{\delta}(t)$ starts to deviate from the classical fidelity $F_{\delta}^{\mathrm{cl}}(t)$ which continues to decay with Lyapunov rates for chaotic systems. On the other hand, for $t \gg t^{*}$, one can use the correlation function formula (12), or Fermi golden rule, to predict decay of quantum fidelity, which now decays with a slower rate (for $\sigma<1$ ) depending quadratically on the strength of perturbation.

Quite paradoxically, for $t>t^{*}$, the dependence on the strength of chaos of $F_{\delta}(t)$ and $F_{\delta}^{\mathrm{cl}}(t)$ is just the opposite than for $t<t^{*}$. While for $t<t^{*}$ both objects (quantum and classical) follow intuitive behavior (more chaos lower fidelity), on the contrary for $t>t^{*}$ the quantum fidelity $F_{\delta}(t)$ behaves just the opposite in the sense (more chaos - faster decay of temporal correlations - higher fidelity).

In order to illustrate all these behaviors in as intuitive way as possible we provided cartoons in the classical phase space of Wigner functions and classical densities, and the corresponding fidelities. For the purpose of numerical demonstration we choose a kicked top [10], a system of a single quantum spin $\vec{J}$ with time-dependent Hamiltonian

$$
H_{\delta}(\vec{J}, t)=(\alpha+\delta) \frac{J_{z}^{2}}{2 J^{2}}+\gamma \sum_{k=-\infty}^{\infty} \delta(t-k) \frac{J_{y}}{J},
$$

and we fix $\hbar=1 / J=0.01$. Perturbation is chosen simply as a variation of the parameter $\alpha$ with the strength $\delta=0.04$, while the parameter $\gamma$ is fixed to $\gamma=\pi / 2$. The classical phase space is now a unit sphere $\vec{j}, \vec{j} \cdot \vec{j}=1$, which we parameterize with two spherical angles $\varphi, \vartheta \in[0,2 \pi] \times[0, \pi]$, as $j_{\mathrm{x}}=\sin \vartheta \cos \varphi$, $j_{\mathrm{y}}=\sin \vartheta \sin \varphi, j_{\mathrm{z}}=\cos \vartheta$. We chose two different values of the parameter alpha: $\alpha=1$ where the corresponding classical dynamics is almost regular (KAM), and $\alpha=4$ where the corresponding classical dynamics is predominantly chaotic almost ergodic. We compute the Wigner function $W_{\psi}(\varphi, z)$, where $z=\cos \vartheta$, of a quantum state of angular momentum using a prescription due to Agarawal [11]. Note that $(\varphi, z)$ are canonical coordinates in the sense of preservation of phase space area under dynamics.

As for the initial state we choose the most classical state, namely $\mathrm{SU}(2)$ coherent state $\left|\vartheta^{*}, \varphi^{*}\right\rangle$ centered at the point $\left(\varphi^{*}, z^{*}=\cos \vartheta^{*}\right)$ in the classical phase space

$$
\begin{aligned}
\left|\vartheta^{*}, \varphi^{*}\right\rangle & =\sum_{m=-J}^{J}\left(\begin{array}{c}
2 J \\
J+m
\end{array}\right)^{1 / 2} \cos ^{J+m}\left(\frac{\vartheta^{*}}{2}\right) \\
& \times \sin ^{J-m}\left(\frac{\vartheta^{*}}{2}\right) e^{-i m \varphi^{*}}|m\rangle .
\end{aligned}
$$

where $|m\rangle$ are eigenstates of $J_{\mathrm{z}}$. Note that the Wigner functions of coherent states - which we shall take as initial Louivillian densities for the corresponding classical dynamics are positive almost-Gaussian distributions centered around $\left(\varphi^{*}, z^{*}\right)$.

First, let us consider the classically chaotic case $\alpha=4$. In fig. 1 we compare time evolutions of the quantum Wigner function and the classical Liouville density starting from the same (nearly) Gaussian initial wave packet. In addition we show the evolution of perturbed dynamics, namely the quantity $W_{U(t) \psi}\left(W_{U(t) \psi}-W_{U_{\delta}(t) \psi}\right)$ and the corresponding classical analog, whose phase space integrals yield, correspondingly, the quantum and the classical fidelity. It is interesting to note that around the Ehrenfest time $t^{*}$, the Wigner function starts to exhibit oscillatory behavior due to interference fringes and thus its structure starts to deviate qualitatively from the Liouville density which remains strictly positive but at the same time $t_{*}$ starts to develop phase-space structures on a scale smaller than $\hbar$. Even further, the value distribution of the 


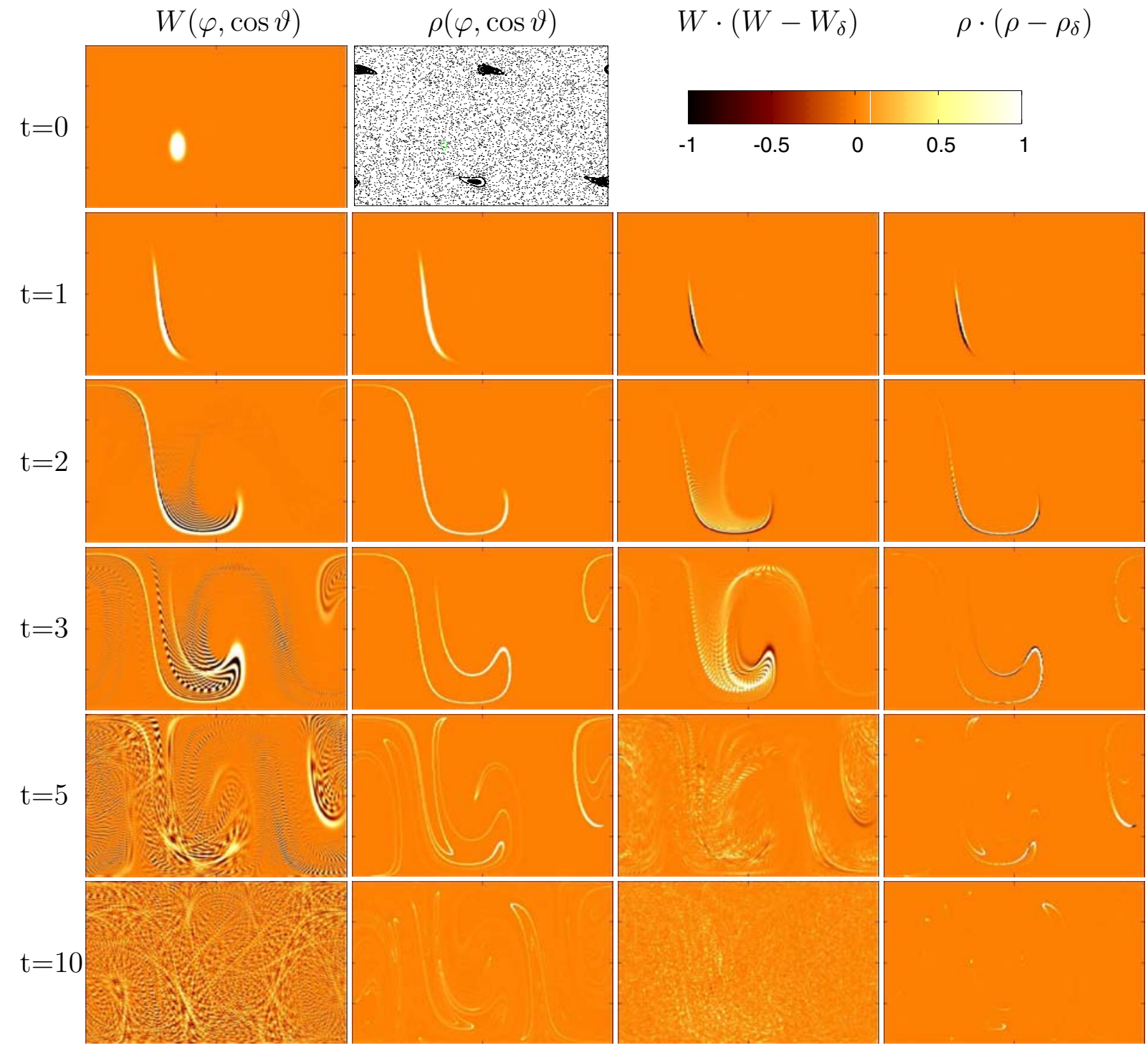

FIG. 1: Wigner function against classical Liouville density for chaotic dynamics $\alpha=4$ of the kicked top, as a function of time, for $t=$ $0,1,2,3,5,10$ kicks. Rectangular phase space $(\varphi, \cos \vartheta) \in[0,2 \pi] \times[-1,1]$ parameterizes the unit sphere. Both densities start from a nearlyGaussian packet corresponding to a coherent state sitting at $\varphi^{*}=\pi / \sqrt{2}, \vartheta^{*}=\pi / \sqrt{3}$. Second frame on the top shows the corresponding classical phase space portrait. The first column is the quantum Wigner function, the second column is the corresponding classical Liouville density, the third and the fourth columns are the corresponding quantum and classical quantities which upon integration give the fidelities. Color (grey) scale indicates the density value (dark=negative, bright=positive).

Wigner function for such an increasingly random state approaches a Gaussian and it's nodal line/domain structure resembles that of an eigenfunction of a Laplacian in a 'chaotic' billiard domain [9]. This is illustrated by zoom-in of the Wigner function and Liouville density evolutions in Fig. 2.

In Fig. 3 we show the corresponding curves of quantum and classical fidelity as a function of time. We note that up to the Ehrenfest time $t^{*}$, which is in this case around 3 kicks, the two quantities agree, and after that they strongly deviate. After $t^{*}$ one can argue that the double integral of time-correlation function (13) in formula (12) starts to behave asymptotically as $\sim \sigma t$ where $\sigma \propto \delta^{2}$ is a certain diffusion constant. This is consistent with oscillating behavior of the Wigner function, which is necessary in order to prevent faster - Lyapunov decay as it happens for the classical fidelity [8]. In a semi-log scale (Fig. 4) one can clearly see asymptotic exponential decays, for the quantum curve the rate is determined by Fermi Golden rule and for the classical curve by the Lyapunov exponent. The former scales with perturbation strength as $\propto \delta^{2}$ while the latter is $\delta$-independent. This a clear manifestation of the qualitative breakdown of quantum-classical correspondence at the Ehrenfest (log-time) barrier $t^{*}$. Beyond $t^{*}$ quantum and classical fidelities behave just in the opposite ways with respect to a degree of chaoticity of the underlying classical system.

Due to unitarity of quantum and classical time-evolution, one can use the equivalence between fidelity and the 


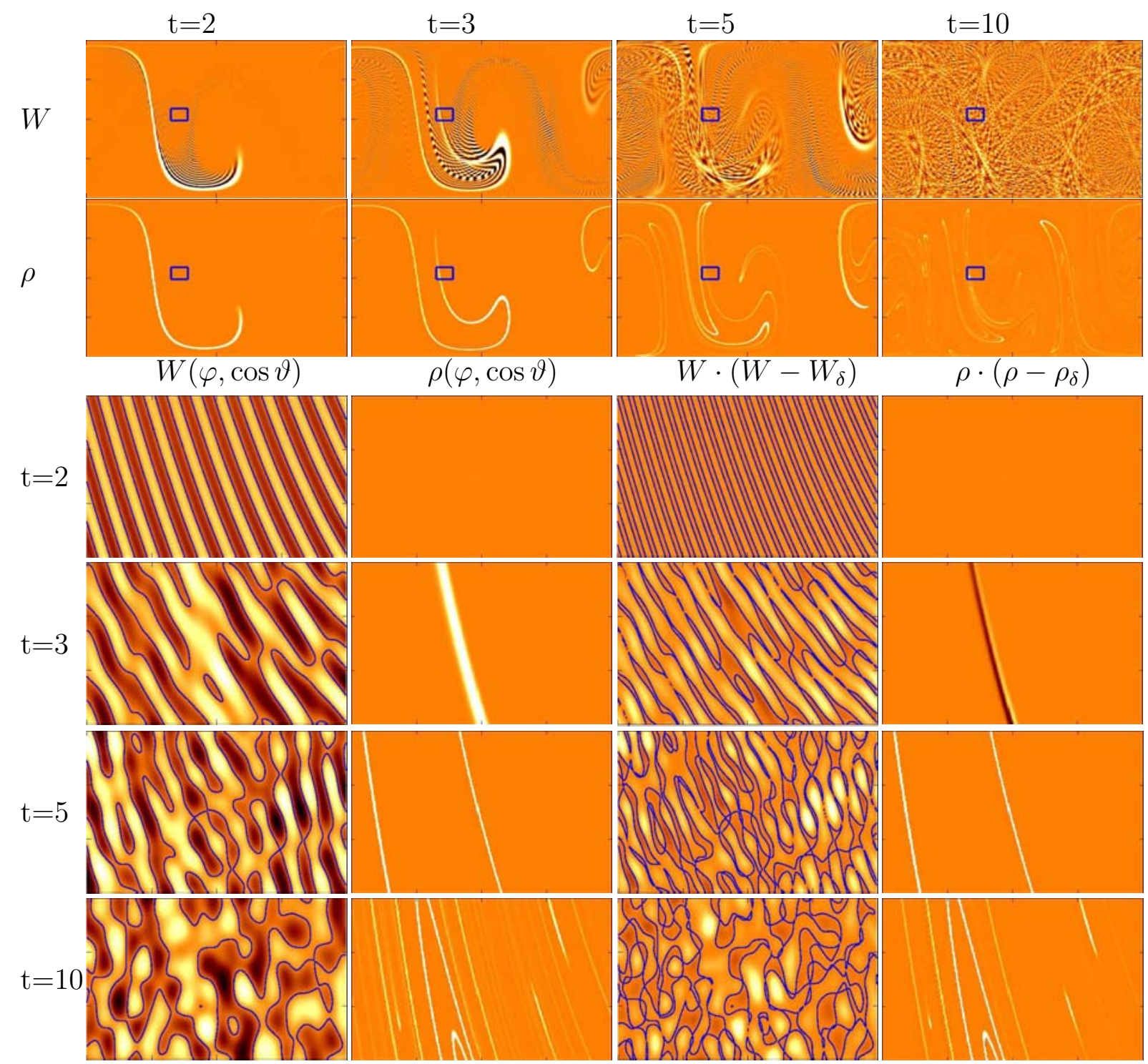

FIG. 2: Zoom-in of Wigner function against classical Liouville density for chaotic dynamics $\alpha=4$. A region of the phase-space area of one Planck cell $2 \pi \hbar$ (indicated above by small rectangles) is magnified in snapshots below. Again, the same representation is used as in fig. 1 . Curves in Wigner function plots are nodal lines.

Loschmidt echo, namely equivalence between representations $(16,22)$ and $(17,23)$. Therefore, fidelity of an initial Gaussian wave-packet (coherent state) can be computed as an overlap of the initial Gaussian with the Wigner function (Liouville density) after echo-dynamics. In fig. 5 we show the corresponding echoed Wigner functions and echoed Lioville densities, which again exhibit significant deviation (due to oscillatory behavior in the Wigner function) at around $t^{*}$.

Second, for completing the story, we make the same numerical experiments in the regime where the classical dynamics is quasi-regular, $\alpha=1$. Since here we have a linear instead of an exponential divergence of classical packets, one expects the breakdown time to scale as $t^{*} \propto \hbar^{-1 / 2}$. In fig. 6 we show an analogous plot to fig. 1. Note that quantum Wigner function and classical Liouville density for short times are here hardly distinguishable in contrast to classically chaotic dynamics. This fact is also illustrated by plotting in fig. 7 , in comparison, quantum and classical fidelity curves for the regular case.

\section{RELATION TO DECOHERENCE MEASURES AND CONCLUSIONS}

Understanding of the behavior of quantum fidelity or quantum Loschmidt echoes, as discussed in previous sections, may turn useful for a design of devices that will be capable of manipulation with quantum information (quantum computers), and to understand the origin of dynamical irreversibility in statistical Hamiltonian mechanics. 


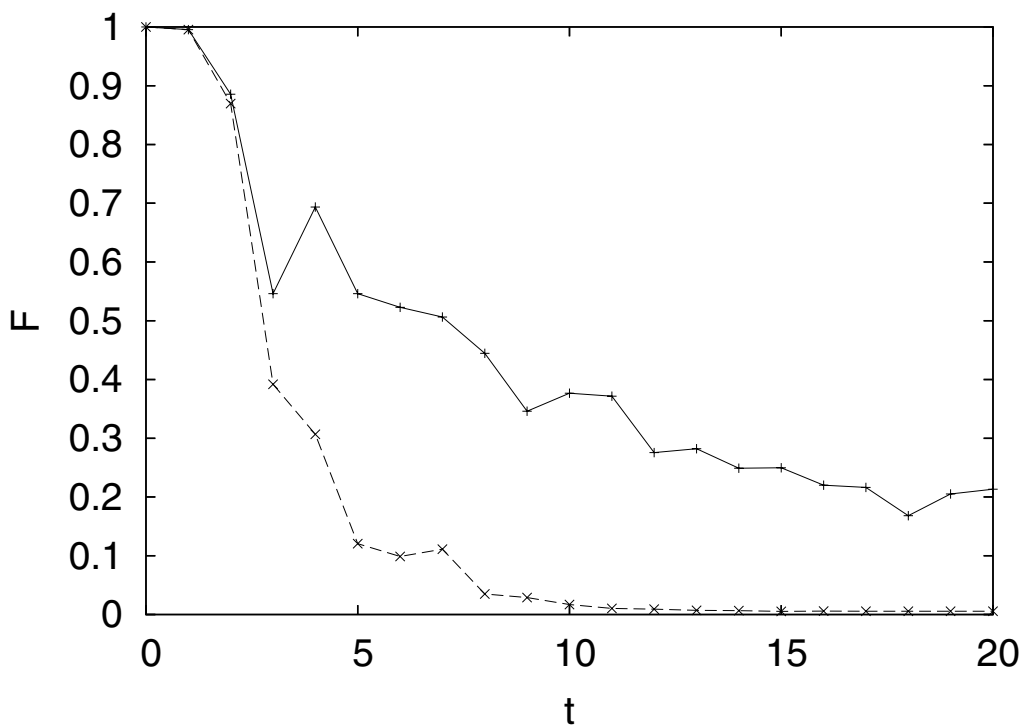

FIG. 3: Quantum fidelity (full curve) against classical fidelity (dashed) for classically fully chaotic dynamics, $\alpha=4$. We observe clear deviation of the two curves at the log-time (or Ehrenfest time) $t^{*} \approx 3$.

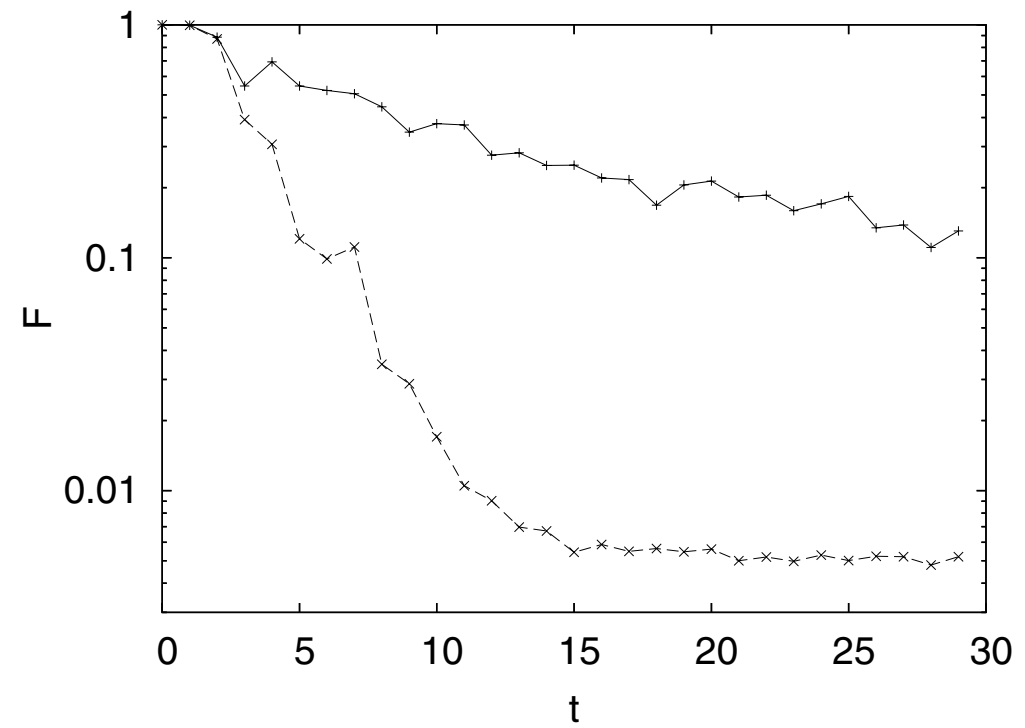

FIG. 4: The same as in previous figure (3) but in semi-log scale. One may observe exponential decays.

In addition, there is also a link between fidelity and decoherence as expressed in terms of a purity of a reduced density matrix. Let us assume that we want to describe a composite quantum system, which is a direct product of the central system $\mathcal{H}_{\mathrm{c}}$ and the environment $\mathcal{H}_{\mathrm{e}}$, using a unitary quantum evolution on the product Hilbert space $\mathcal{H}_{\mathrm{c}} \times \mathcal{H}_{\mathrm{e}}$. Decoherence is then directly related to the loss of purity of reduced density matrix $\rho_{\mathrm{c}}(t)=\operatorname{tr}_{\mathrm{e}}|\psi(t)\rangle\langle\psi(t)|$. The purity is quantified as $I(t)=\operatorname{tr}_{\mathrm{c}}\left[\rho_{\mathrm{c}}(t)\right]^{2}$ and is equal to 1 for pure states and $\sim 1 / N$ for maximally mixed states, where $N$ is the dimension of the smaller of the Hilbert spaces $\mathcal{H}_{\mathrm{c}}$, and $\mathcal{H}_{\mathrm{e}}$. The rate of decoherence can thus be measured in terms of decay of $I(t)$ down from 1 .
One can show a strict inequality between purity $I(t)$ and quantum fidelity $F(t)$ as defined for evolving the same initial state with two dynamics: the coupled system (central system Hamiltonian plus environment Hamiltonian plus coupling) as compared to the uncoupled system (total Hamiltonian without the coupling). Namely the strong result [12] shows that

$$
I(t) \geq(F(t))^{2} .
$$

Thus the understanding of the decay rates of fidelity provides the upper bounds to the decay rates for purity. In fact, it has been shown [12] that this upper bound is typically reached (equality sign in (26)) in the case of chaotic classical dynamics, whereas in classically regular dynamics the purity may 


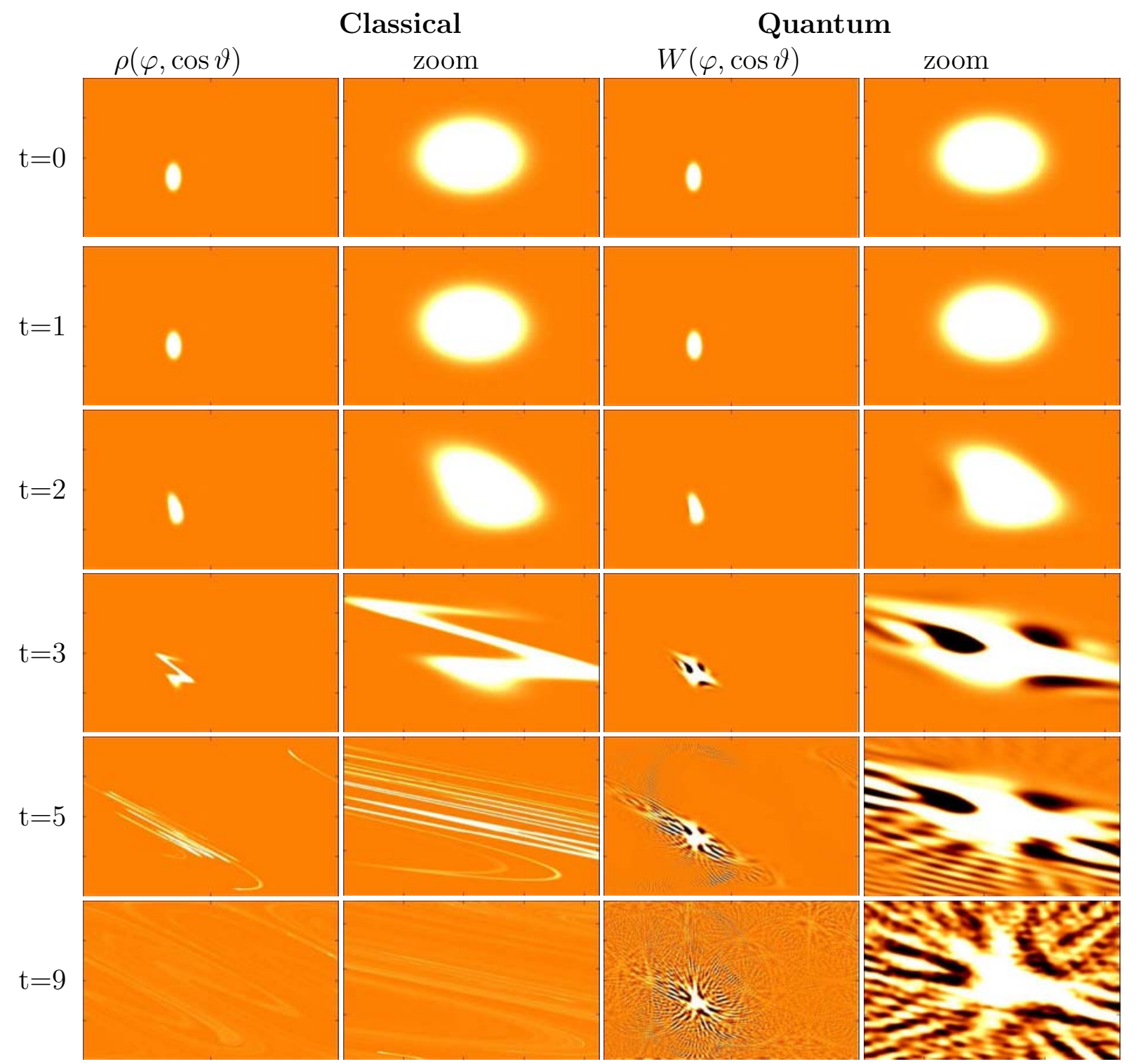

FIG. 5: Wigner function and classical densities of the echo-dynamics of classically chaotic dynamics. Quantum (first column, magnified in second column) against classical (third column, magnified in fourth column). All the parameters are the same as in fig. 1.

decay drastically slower [13].

In this paper we have reviewed recent theoretical approaches to describe decay of quantum fidelity in dynamical systems, and in addition provided clear phase space picture and illustration of the approach in terms of quantum Wigner functions. It is clear that seemingly paradoxical behavior of quantum fidelity with respect to the classical correspondent is a consequence of the breakdown of quantum classical correspondence in the Wigner function at the Ehrenfest time.

\section{ACKNOWLEDGMENTS}

We thank T.H.Seligman and G.Casati for useful discussions. The work has been financially supported by the grant P1-044 of the Ministry of Education, Science and Sports of Slovenia, and in part by the ARO grant (USA) DAAD 19-02$1-0086$.
[1] R. A. Jalabert and H. M. Pastawski, Phys. Rev. Lett. 86, 2490 (2001).

[2] T. Prosen, Phys. Rev. E 65, 036208 (2002).
[3] Ph. Jacquod, P. G. Silvestrov, and C. W. J. Beenakker, Phys. Rev. E 64, 055203 (2001).

[4] T. Prosen and M. Žnidarič, J. Phys. A: Math. Gen 35, 1455 


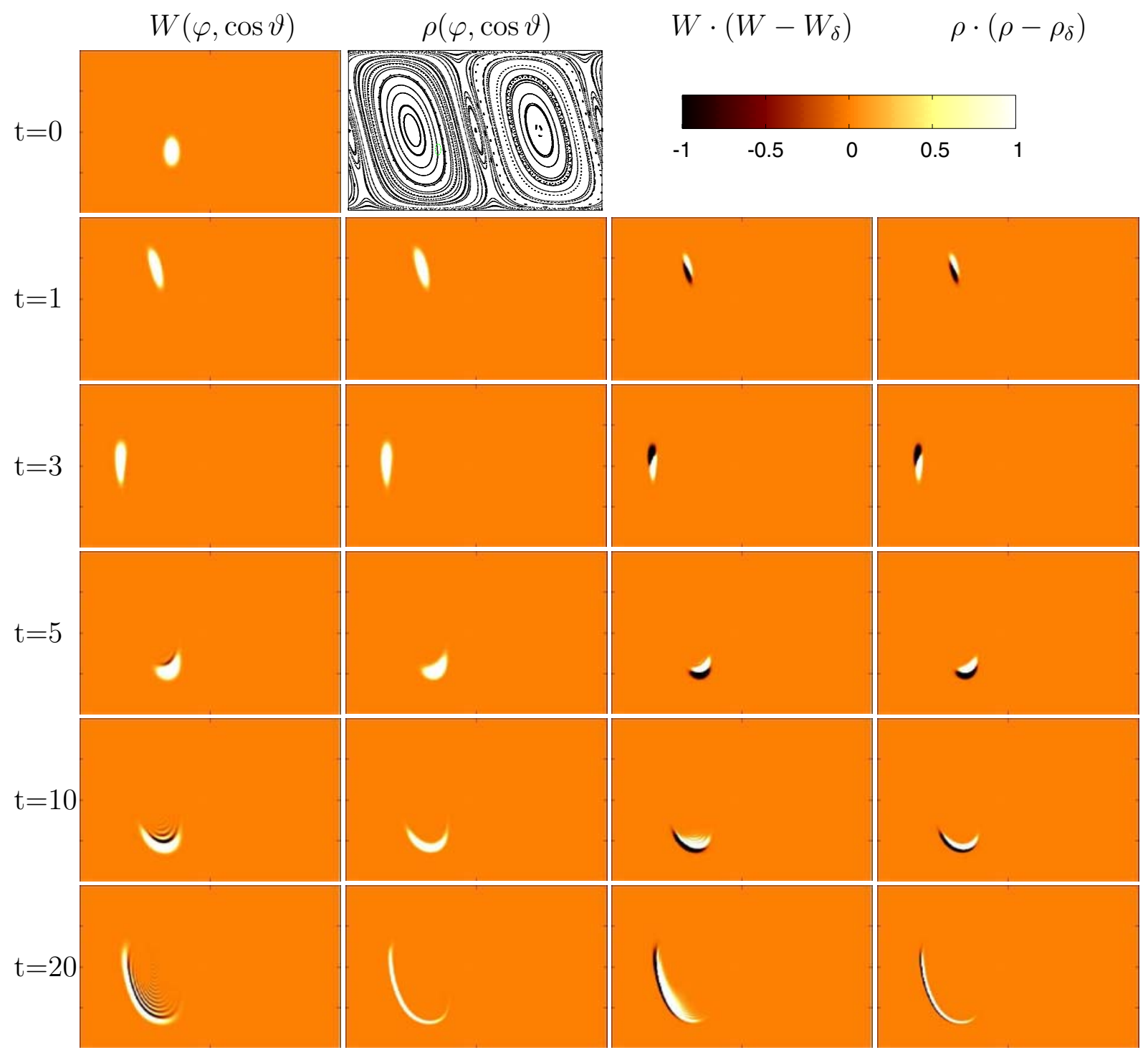

FIG. 6: Wigner function against classical density evolutions for regular dynamics. Everything is the same as in fig.1 except now for $\alpha=1$.

(2002).

[5] F. M. Cucchietti, H. M. Pastawski, and D. A. Wisniacki, Phys. Rev. E 65, 045206(R) (2002); S. Tomsovic and N. Cerruti, Phys. Rev. Lett. 88, 054103 (2002); G. Benenti and G. Casati, Phys. Rev. E 66, 066205 (2002); F. M. Cucchietti et al. Phys. Rev. E 65, 046209 (2002); D. A. Wisniacki et al. Phys. Rev. E 65, 055206 (2002); D. A. Wisniacki and D. Cohen, Phys. Rev. E 66, 046209 (2002); J. Emerson et al, Phys. Rev. Lett. 89, 284102 (2002); G. P .Berman et al, Phys. Rev. E 66, 056206 (2002); W. G. Wang and Baowen Li, Phys. Rev. E 66, 056208 (2002); T. Kottos and D. Cohen, Europhys. Lett. 61, 431 (2003); D. A. Wisniacki, Phys. Rev. E 67, 016205 (2003); P. G. Silvestrov, J. Tworzydlo, and C. W. J. Beenakker, Phys. Rev. E. 67, 025204 (2003); and many other references.

[6] H. M. Pastawski, P. R. Levstein, and G. Usaj,
Phys. Rev. Lett.75, 4310 (1995); P. R. Levstein, G. Usaj, and H. M. Pastawski, J. Chem. Phys. 108, 2718 (1998).

[7] A. Peres, Phys. Rev. A 30, 1610 (1984).

[8] G. Veble and T. Prosen, Phys. Rev. Lett. 92, 034101 (2004).

[9] M. Horvat and T. Prosen, J. Phys. A: Math. Gen. 36, 4015 (2003).

[10] F. Haake, M. Kuś, and R. Schar, Z. Phys. B 65, 381 (1987).

[11] G. S. Agarwal, Phys. Rev. A 24, 2889 (1981).

[12] M. Žnidarič, and T. Prosen, J. Phys. A: Math. Gen. 36, 2463 (2003); see also T. Prosen, T. H. Seligman, and M. Žnidarič, Phys. Rev. A 67, 062108 (2003). 042105 (2004).

[13] M. Žnidarič and T. Prosen, Phys. Rev. A 71, 032103 (2005). 


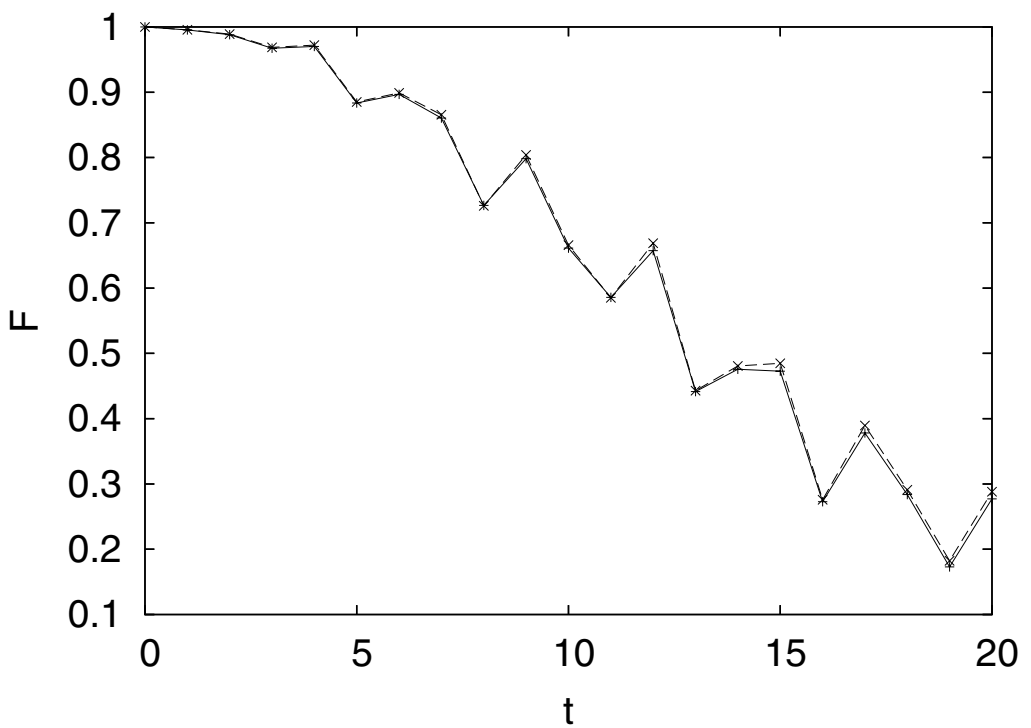

FIG. 7: Quantum fidelity (full curve) against classical fidelity (dashed) for classically regular dynamics $\alpha=1$. Other parameters are the same as in fig. 3 . 